\title{
Electric-field-driven Mott metal-insulator transition in correlated thin films: An inhomogeneous dynamical mean-field theory approach
}

\author{
P. Bakalov, ${ }^{1, *}$ D. Nasr Esfahani, ${ }^{2,3, \dagger}$ L. Covaci, ${ }^{2, \dagger}$ F. M. Peeters, ${ }^{2, \S}$ J. Tempere,,${ }^{4, \mathbb{I}}$ and J.-P. Locquet ${ }^{1, * *}$ \\ ${ }^{1}$ Departement Natuurkunde en Sterrenkunde, KULeuven, Celestijnenlaan 200D, B-3001 Leuven, Belgium \\ ${ }^{2}$ Departement Fysica, Universiteit Antwerpen, Groenenborgerlaan 171, B-2020 Antwerpen, Belgium \\ ${ }^{3}$ Condensed Matter National Laboratory, Institute for Research in Fundamental Sciences (IPM), Tehran 19395-5531, Iran \\ ${ }^{4}$ Departement Fysica, Universiteit Antwerpen, Universiteitsplein 1, B-2610 Wilrijk, Belgium
}

(Received 23 February 2015; revised manuscript received 16 March 2016; published 8 April 2016)

\begin{abstract}
Simulations are carried out based on the dynamical mean-field theory (DMFT) in order to investigate the properties of correlated thin films for various values of the chemical potential, temperature, interaction strength, and applied transverse electric field. Application of a sufficiently strong field to a thin film at half filling leads to the appearance of conducting regions near the surfaces of the film, whereas in doped slabs the application of a field leads to a conductivity enhancement on one side of the film and a gradual transition to the insulating state on the opposite side. In addition to the inhomogeneous DMFT, a local density approximation (LDA) is considered in which the particle density $n$, quasiparticle residue $Z$, and spectral weight at the Fermi level $A(\omega=0)$ of each layer are approximated by a homogeneous bulk environment. A systematic comparison between the two approaches reveals that the less expensive LDA results are in good agreement with the DMFT approach, except close to the metal-to-insulator transition points and in the layers immediately at the film surfaces. LDA values for $n$ are overall more reliable than those for $Z$ and $A(\omega=0)$. The hysteretic behavior (memory effect) characteristic of the bulk doping driven Mott transition persists in the slab.
\end{abstract}

DOI: 10.1103/PhysRevB.93.165112

\section{INTRODUCTION}

Due to their interesting and useful properties, strongly correlated materials have attracted the attention of both theorists [1-3] and experimentalists [4-8] in recent decades. In particular, the discoveries of high-temperature superconductivity in cuprates and of colossal magnetoresistance in manganites spurred interest in the class of materials exhibiting the Mott metal-to-insulator transition $[9,10]$. The existence of this correlation-driven transition in bulk transition-metal oxide systems has encouraged investigations for potential applications in electronics [5-7]. Intriguing physics has also been observed at interfaces between strongly correlated materials [11]; for example, a metallic or even superconducting phase appears at the interface between $\mathrm{LaTiO}_{3}$ and $\mathrm{SrTiO}_{3}$, which are insulating paramagnets in bulk $[8,12]$. The appearance of such interface phases can be partially understood at a qualitative level as the result of charge transfer. A detailed understanding, however, remains elusive. Due to the difficulties inherent in the theoretical treatment of strongly-correlated systems, attaining a detailed understanding may be quite challenging even when dealing with simpler bulk systems.

The exponential growth of the Hilbert space with the number of particles makes a direct numerical solution of an interacting quantum system unfeasible, except for systems with a very limited number of particles. Standard theoretical

\footnotetext{
*petar.bakalov@fys.kuleuven.be

†Davoud.NasrEsfahani@uantwerpen.be

†lucian@covaci.org

§Francois.Peeters@uantwerpen.be

Ijacques.tempere@uantwerpen.be

***jeanpierre.locquet@fys.kuleuven.be
}

approaches such as density functional theory (DFT) are known to fail when electron-electron interactions are strong [13]. This has stimulated the development of alternative methods. The Gutzwiller approximation [14-16] and slave boson techniques $[17,18]$ have been used to treat the low-energy physics of bulk materials and - more recently - thin films [19-24] in the presence of an electric field [21,22]. The slave boson approach has been extended to allow access to higher-energy excitations [25], while the Gutzwiller approximation only provides insight into the metallic state. Other notable approaches include the density renormalization group (DMRG), which works best for one-dimensional systems [26]. Over the last 25 years dynamical mean-field theory (DMFT) has emerged as one of the most promising frameworks for the treatment of strongly correlated systems [27,28]. DMFT involves a mapping of a lattice problem, such as the Hubbard model [29], onto an interacting quantum impurity model [30] supplemented by a self-consistency condition. The mapping is exact in the limit of infinite dimensions. An advantage of DMFT is that it is formulated in the thermodynamic limit and that it allows one to nonperturbatively interpolate between the strong and weak coupling regimes, thereby treating different interaction regimes on an equal footing. In its simplest version, single-site DMFT, it neglects spatial correlations, but captures local temporal correlations. In combination with the local density approximation (LDA) to DFT it has provided valuable new insights into the physics of a number of strongly correlated bulk materials $[13,31,32]$.

A better understanding of the metal-insulator transition in systems with lower translational symmetry (such as thin films or interfaces) and in the presence of an electric field is likely to aid experimental efforts to control the properties of such systems, which is relevant for possible applications in, e.g., memory devices and high-speed electronics. Potthoff and 
Nolting [33,34], and later others [35-43], used a spatially resolved version of DMFT (inhomogeneous DMFT, or IDMFT) to study layered systems. Similar techniques were later applied to inhomogeneous multilayered nanostructures $[1,2,44,45]$. DMFT-based approaches were recently proposed for the study of "Mott $p$ - $n$ junctions" [46] and correlated capacitors [47]. In these approaches each layer was approximated by a twodimensional bulk system with the appropriate value of the chemical potential, and a Poisson solver was used to study the charge redistribution in different regimes.

In some of these earlier studies [40,41] Okamoto used a combination of DMFT with the Keldysh Green's function approach to study the transport properties of a system of Hubbard-interacting layers coupled to metallic leads and found that such structures exhibit strongly nonlinear current-voltage characteristics. When the applied bias was comparable to the on-site interaction $U$, Okamoto also observed the formation of quasiparticlelike features in the spectrum of the correlated part of the system.

Other studies focused on interacting heterostructures in the static limit (i.e., zero current) and employed the spinless Falicov-Kimball model for the interaction [44]. This model, which involves interactions between spinless conduction electrons with spinless localized electrons and exhibits a Mottlike metal-to-insulator transition, allows extremely accurate numerical solutions [44], but—unlike the Hubbard model—is not a Fermi liquid in the metallic phase.

In this paper we use the IDMFT to investigate the effect of an applied electric field on the properties of a slab (a stack of coupled Hubbard-correlated two-dimensional layers) at various temperatures and doping levels in the static limit. We also approximate the properties of each layer in the slab by a three-dimensional bulk system in which the value of the chemical potential is equal to the local value of the potential in that layer. We refer to this approach as the local density approximation, a name which is used in the cold atom community (not to be confused with the LDA used in the context of DFT), and we carry out a systematic comparison between the LDA and the full DMFT calculation. This comparison is of some interest, since the LDA is significantly faster than the full calculation. We investigate the effect of screening by considering the Coulomb interaction between planes in the slab at a mean-field level.

The structure of this paper is as follows. We describe the model and method in Sec. II. Our results are discussed in Sec. III. Section IV provides a summary.

\section{MODEL AND METHOD}

The general approach that we use is described in detail elsewhere $[33,34]$, and we only give a brief description here. Our starting point is the single-band Hubbard Hamiltonian:

$$
\begin{aligned}
H= & H_{\mathrm{kin}}+H_{U}+H_{\mathrm{CP}} \\
= & -\sum_{i j \gamma \delta \sigma} t_{\gamma \delta}^{i j} c_{i \gamma \sigma}^{\dagger} c_{j \delta \sigma}+U \sum_{i \gamma} n_{i \gamma \downarrow} n_{i \gamma \uparrow} \\
& -\mu \sum_{i \gamma \sigma} n_{i \gamma \sigma},
\end{aligned}
$$

where $c_{i \gamma \sigma}^{\dagger}\left(c_{i \gamma \sigma}\right)$ is a fermionic creation (annihilation) operator for a particle of $\operatorname{spin} \sigma(\sigma=\uparrow, \downarrow)$ at site $\gamma$ in plane $i, n_{i \gamma \sigma}=c_{i \gamma \sigma}^{\dagger} c_{i \gamma \sigma}, t_{\gamma \delta}^{i j}$ is a hopping matrix, $U$ is the on-site Coulomb repulsion, $\mu$ is the chemical potential. We work with a simple cubic lattice and assume that only nearest-neighbor hopping takes place, and that the intra- and interplane hopping parameters are equal, i.e., all hopping matrix elements vanish except $t_{\langle\gamma \delta\rangle}^{i i}=t_{\gamma \gamma}^{i i \pm 1} \equiv t$, where the angular brackets indicate that sites $\gamma$ and $\delta$ are next neighbors. We take $t=1$, which sets the energy scale in the problem. In order to include the effect of an externally applied electric field, we include the following term:

$$
H_{\mathrm{EP}}=\sum_{i \gamma \sigma} v_{i} n_{i \gamma \sigma},
$$

where $v_{i}=-V(i /(L-1)-1 / 2)$ is the on-site potential for plane $i$ ( $V$ is the overall potential drop across the slab and $L$ is the number of planes in the slab; $L=24$ throughout this paper). This form assumes that the charge in the slab does not screen the externally applied field and neglects any Coulomb interaction between the layers themselves. We also include the effects of screening by including a correction to the bare potential of the form $[46,48]$ :

$$
v_{\text {Coulomb }, \mathrm{i}}=\alpha \sum_{j \neq i}\left(n_{j}-n_{\text {bulk }}\right)|i-j|,
$$

where $n_{\text {bulk }}$ is the number of electrons per site in the neutral solid, $n_{j}=n_{j, \uparrow}+n_{j, \downarrow}$ is the average number of electrons at sites in plane $j$ (site index $\gamma$ is suppressed, since density does not vary within a plane), and $\alpha$ determines the interaction strength between charges, $\alpha=\frac{e^{2} d}{2 \epsilon_{r} \epsilon_{0} A}$. In the last expression $e$ is the elementary charge, $\epsilon_{r}$ is the relative dielectric constant of the solid, $d$ is the distance between planes in our thin film, and $A$ is the area per atom within each plane. For example, taking $d=10^{-9} \mathrm{~m}, A=d^{2}$, and $\epsilon_{r}=10$, one has $\alpha \approx 2 \mathrm{eV}$. The approximate form, Eq. (3), corresponds to the assumption that the charge in each plane is uniformly distributed. It is used here for simplicity, as it is likely to capture the qualitative effects of screening (more accurate forms have also been used previously [36-39,43]). Moreover, it is in qualitative agreement with recent calculations based on the constrained random phase approximation in few-layer $\mathrm{SrVO}_{3}$ systems [49].

In the absence of screening, we use the standard DMFT loop: Starting from a guess for the slab self-energy, $\hat{\Sigma}_{\sigma}\left(i \omega_{n}\right)=$ $\operatorname{Diag}\left(\Sigma_{\sigma 1}\left(i \omega_{n}\right), \Sigma_{\sigma 2}\left(i \omega_{n}\right), \ldots\right)$, we obtain the bare Green's function (also referred to as the Weiss field) of the effective action for each layer in the slab, $\mathcal{G}_{0, i \sigma}\left(i \omega_{n}\right)$ :

$$
\mathcal{G}_{0, i \sigma}^{-1}\left(i \omega_{n}\right)=\left[G_{i i \sigma}\left(i \omega_{n}\right)\right]^{-1}+\Sigma_{\sigma i}\left(i \omega_{n}\right),
$$

where $\omega_{n}=(2 n+1) \pi / \beta$ are the fermionic Matsubara frequencies at temperature $T=1 / \beta$, the index $i=1,2, \ldots L$, and $G_{i i \sigma}$ is a diagonal element of the $k$-integrated slab Green's function corresponding to $\hat{\Sigma}_{\sigma}\left(i \omega_{n}\right)$ :

$$
\hat{G}_{\sigma}\left(i \omega_{n}\right)=\frac{1}{N_{k}} \sum_{\mathbf{k} \in B Z} \frac{\mathbf{1}}{\left(i \omega_{n}+\mu\right) \mathbf{1}-\hat{H}_{\sigma}(\mathbf{k})-\hat{\Sigma}_{\sigma}\left(i \omega_{n}\right)},
$$

where 1 is a $L \times L$ unit matrix ( $L$ is the number of layers in the slab), $N_{k}=N_{k_{x}} \times N_{k_{y}}, N_{k_{x}}$ and $N_{k_{y}}$ are the number 
of $k$ points in the $x$ and $y$ directions of the Brillouin zone, respectively, and $\hat{H}_{\sigma}(\mathbf{k})$ is a tridiagonal $L \times L$ matrix in a mixed (Fourier/real-space) basis obtained from the sum of $H_{\text {kin }}$ and $H_{\mathrm{EP}}$ :

$$
\hat{H}_{\sigma}(\mathbf{k})=\left(\begin{array}{cccc}
\epsilon_{\mathbf{k}}+v_{1} & -t & 0 & \cdots \\
-t & \epsilon_{\mathbf{k}}+v_{2} & -t & \cdots \\
0 & -t & \epsilon_{\mathbf{k}}+v_{3} & \cdots \\
\vdots & \vdots & \ddots &
\end{array}\right),
$$

$\epsilon_{\mathbf{k}}=-2 t\left(\cos k_{x}+\cos k_{y}\right)$ being the in-plane dispersion. The impurity Green's function for each layer, $G_{\mathrm{imp}, i \sigma}\left(i \omega_{n}\right)$, is obtained by solving the respective impurity problem determined by $\mathcal{G}_{0, i \sigma}$. From the impurity Green's function a new $\Sigma_{\sigma i}\left(i \omega_{n}\right)$ is obtained using Eq. (4) with $G_{i i \sigma}$ replaced by $G_{\mathrm{imp}, i \sigma} . \hat{G}_{\sigma}\left(i \omega_{n}\right)$ is only recalculated once the solver has swept through all the layers and all $\hat{\Sigma}_{\sigma i}$ are updated. This is repeated until convergence.

To include screening, we adjust the potential according to Eq. (3) after each solver sweep [just before the recalculation of $\left.G_{\text {imp }, i \sigma}\left(i \omega_{n}\right)\right]$. For the doped calculations (not screened) it is also necessary to adjust the chemical potential of the slab between solver sweeps in order to keep the density fixed.

To solve the resulting DMFT equations we use the hybridization expansion continuous-time quantum Monte Carlo solver provided as a part of TRIQS (a toolbox for research on interacting quantum systems) [50-53]. In the segment picture [52] the on-site densities are obtained directly from the solver. We estimate the quasiparticle weight $Z=$ $(1-\partial \operatorname{Im} \Sigma(i \omega) / \partial \omega)_{\omega \rightarrow 0^{+}}^{-1}$ from the imaginary frequency selfenergy at the lowest Matsubara frequency [54]:

$$
Z \approx\left(1-\frac{\operatorname{Im} \Sigma\left(i \omega_{0}\right)}{\omega_{0}}\right)^{-1}
$$

The simple estimate in Eq. (5) assumes that the imaginary part of the self-energy at zero frequency vanishes, i.e., $\operatorname{Im} \Sigma(\omega=$ $0)=0$. This assumption is not justified in a Fermi liquid at finite temperature (where $\left.\operatorname{Im} \Sigma(\omega=0) \propto T^{2}\right)$ and in the Mott insulating phase (where $\operatorname{Im} \Sigma(\omega=0)$ diverges). This should be kept in mind when interpreting the significance of the $Z$ factor calculated on the basis of Eq. (5). Equation (5) is still useful for presentation purposes, as a proxy of the MIT. For completeness, we include in the Appendix (Sec. A) an estimate for $Z$ based on a polynomial fit to the Matsubara self-energy at the lowest Matsubara frequencies.

To estimate $A(\omega=0)$, the spectral weight at the Fermi level, we use the relation [54]:

$$
\beta G(\beta / 2) \stackrel{\beta \rightarrow \infty}{\longrightarrow}-\pi A(0) .
$$

Aside from the full DMFT calculation described above, we also calculate the expected density distribution in the slab assuming that the density of electrons per site in each layer, $n_{i}$, is determined solely by the local value of the chemical potential and independently of the rest of the layers. Within this approach, we assume that

$$
n_{i}=n_{3 D}\left(\mu_{i}\right),
$$

where $n_{3 D}(\mu)$ is the density per site of the three-dimensional Hubbard model on a cubic lattice with nearest-neighbor hopping at chemical potential $\mu$ and the same values of $U, t$ and $\beta$ as in the slab, and $\mu_{i}=\mu+v_{i} . n_{3 D}(\mu)$ is obtained by single-site DMFT for a set of predetermined values of $\mu$. The density for values of $\mu$ that do not coincide with values in that predetermined set is obtained from a linear interpolation:

$$
n(\mu)=n\left(\mu_{\text {left }}\right) x+n\left(\mu_{\text {right }}\right)(1-x),
$$

where $\mu_{\text {left,right }}$ are the two values of the chemical potential in the set which are closest to $\mu$ (such that $\mu_{\text {left }} \leqslant \mu \leqslant \mu_{\text {right }}$ ), and $x=\left(\mu_{\text {right }}-\mu\right) /\left(\mu_{\text {right }}-\mu_{\text {left }}\right)$. In a similar manner we obtain LDA estimates for the spectral weight at the Fermi level, $A(\omega=0)$, and the $Z$ factor. Two stable solutions of the DMFT equations of the 3D Hubbard model, a metallic and an insulating one, exist in parts of the $U, \beta, \mu$-range which we explore. For the LDA we use the density that corresponds to the metallic branch. In all our calculations we enforce a paramagnetic phase, i.e., we set $\hat{\Sigma}_{\uparrow}=\hat{\Sigma}_{\downarrow}$.

\section{RESULTS}

\section{A. Coexistence}

The dependence of on-site density on chemical potential for the Hubbard model on a three-dimensional tight-binding lattice is shown in Fig. 1 for $U / t=13.2$. For all temperatures shown there is a plateau in the $n(\mu)$ curve. This is due to the existence of a gap in the spectral function of the solid at that value of the Hubbard interaction $U$. When the value of the chemical potential is inside the gap, far from the upper and lower Hubbard bands, the charge density does not change noticeably when the chemical potential is varied, due to the vanishing spectral weight in the gap. As the chemical potential approaches either of the two Hubbard bands, a quasiparticle peak appears at the Fermi level. This redistribution of spectral weight causes a change in the particle density and determines the end of the flat region in the $n(\mu)$ curve.

As is well known, there is a region in the $\mu-U-T$ phase diagram of the three-dimensional fermionic Hubbard model in which both a metallic and an insulating phase can exist $[55,56]$. When the values of $\mu, U$, and $T$ are such that the system is in this coexistence region, the DMFT converges to either one

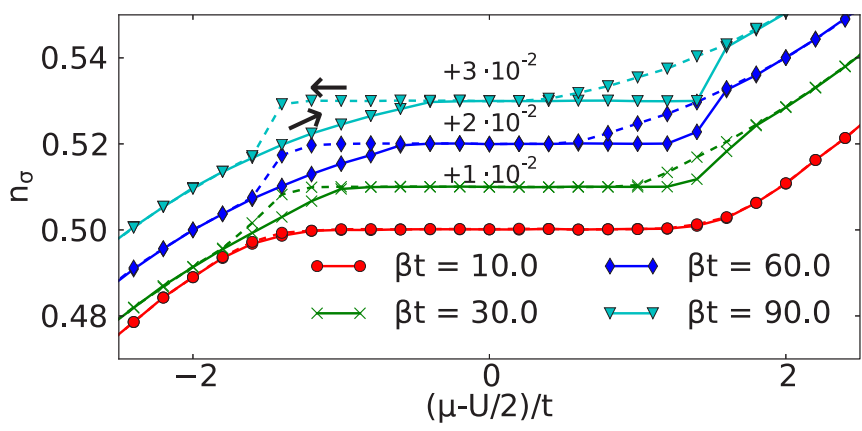

FIG. 1. Bulk (3D) electron density as a function of chemical potential for different temperatures at $U / t=13.2$. This value of the interaction is large enough for a gap to open in the spectral function of the solid. A corresponding plateau appears in the $n(\mu)$ curve. The simulations for different $\mu$ values are carried out recursively. Dashed (full) lines correspond to down- (up-) sweep. The hysteresis indicates the coexistence of two solutions. Offsets added for clarity. 
of the two solutions, depending on the initial guess (seed) for the self-energy. At the value of the Hubbard repulsion that we are considering, $U / t=13.2$, and when the system is far away from half filling (when $\mu-U / 2$ is large), only the metallic solution exists. If one gradually brings the system closer to half filling (by varying $\mu$ in small increments) and uses the converged value of the self-energy at each point as a seed for the subsequent simulation, the system remains on the metallic branch until the metallic solution ceases to exist in the region very close to half filling. Continuing the recursion beyond half filling traces out the insulating branch. This is reflected in the hysteresis in Fig. 1. Coexistence is possible in the slab as well [57].

In Fig. 2(a) we show the variation of the charge density of the metallic solution across the slab calculated with DMFT (symbols) and in the LDA (lines) for a number of different values of the applied external field at $\beta=30$. The interaction strength $(U / t=12)$ is chosen such that the bulk system at half filling $(\mu=U / 2)$ would be in the $U-T$ coexistence region, close to its upper boundary, $U_{c 2}$, beyond which only the insulating phase is stable. A comparison of the two approaches shows that the DMFT and LDA results are in good agreement in the central parts of the slab, whereas near the surface (within approximately the first four layers) the discrepancy is significant. For larger fields the discrepancy between the DMFT and LDA is significant in a narrower region close to the surface. The main reason for this is the existence of highly-doped regions (close to the surfaces) in which the correlation length is smaller [22], which limits the discrepancy between the two approaches to only the surface layer. We expect that the discrepancy between the DMFT and LDA will be larger whenever the correlation length is larger, in particular at very low temperatures and when the first order nature of the transition is weaker. The same trends were observed earlier in calculations based on the Gutzwiller approximation [21].

In Fig. 2(b) we plot the double occupancy, $\left\langle d_{i}\right\rangle=\left\langle n_{i \uparrow} n_{i \downarrow}\right\rangle$, across the slab at zero applied external field for both the metallic and insulating solutions. In both the metallic and the insulating case the double occupancy is suppressed at the surface. The suppression is due to the surface reduction of the site coordination number, which leads to an enhancement
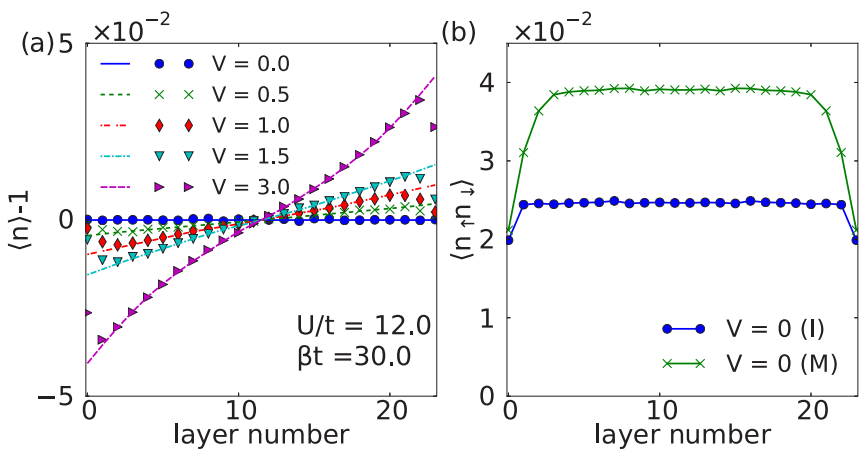

FIG. 2. Charge density deviation from half filling across the slab for different values of the applied field. Symbols indicate the full DMFT calculation; lines correspond to the LDA (panel (a)). Double occupancy across the slab for the insulating and metallic solution at zero field (panel (b)). $U / t=12$ and $\beta t=30$ (both panels). of correlation effects. The surface effect is also visible in the density redistribution in the presence of an electric field [Fig. 2(a)]: The maximum charge deviation does not occur exactly at the surface as is expected from LDA approach. From Fig. 2(b) it is clear that in the insulating state the double occupancy recovers its bulk value within a single layer, whereas in the metallic case the recovery takes place deeper in the slab (approximately four layers from the surface). This suggests that the correlation length in the metallic state is longer than in the insulating state. The recovery length for the double occupancy at $V=0$ in the metallic state is related to the extent of the surface effect in the case of applied electric field, which is given by the width of the discrepancy between the full DMFT calculation and the LDA shown in Fig. 2(a). These two lengths are of approximately the same magnitude.

\section{B. Half-filled case}

The charge redistribution caused by an electric field applied to a slab at half filling is shown in Fig. $3(U / t=13.2)$. The value of $U$ is outside the $U-T$ coexistence region for bulk (at $\mu=U / 2$ ) and is such that the slab is insulating at zero field. As discussed in connection with Fig. 1, the metallic and insulating phase can coexist in bulk at this value of $U$ when $\mu$ is away from its particle-hole symmetric value. The two phases can coexist in the slab as well, and we find that recursive simulations for different values of $V$ lead to different solutions, i.e., hysteresis (not shown). Whenever two distinct solutions exist, in Fig. 3 we show only the metallic one. It is clear from the figure that when the field applied to the slab is below a certain threshold, there is no redistribution of charge in the slab. When the field increases sufficiently, the charge density close to the surfaces of the film starts deviating from half filling. The deviation is symmetric with respect to the center of the slab, as can be expected in the case of a symmetric (with respect to the center of the slab) external field

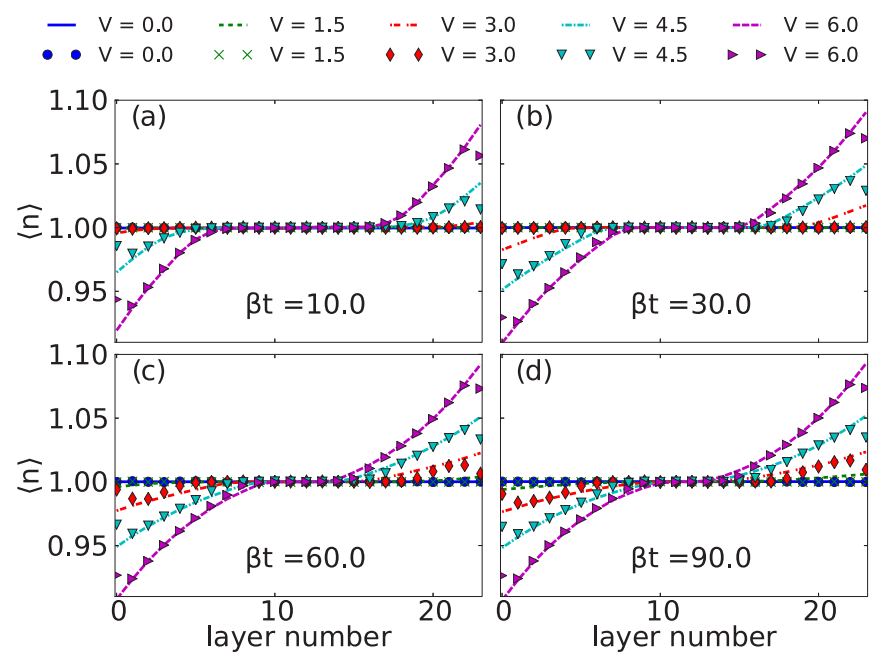

FIG. 3. Electron density across the slab at different temperatures and different values of the electric field. The overall density for the slab corresponds to half filling. Only "metallic" seeds were used. Symbols correspond to the full DMFT calculation, lines correspond to the LDA. $U / t=13.2$. 


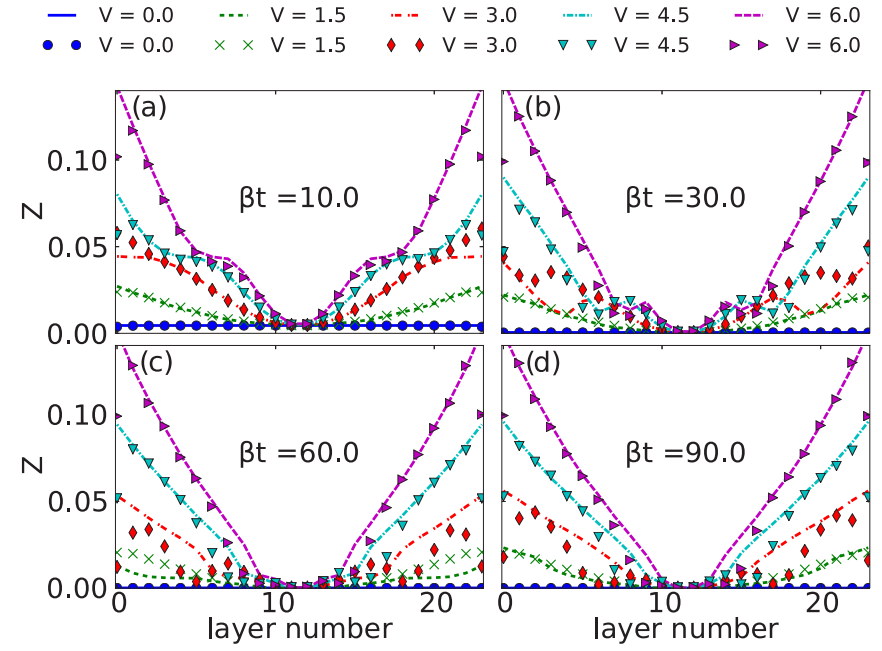

FIG. 4. Variation of the $Z$ factor, Eq. (5), across the slab for the same simulation parameters as in Fig. 3. Symbols correspond to the full DMFT calculation, lines correspond to the LDA.

due to the particle-hole symmetry of the Hubbard model with nearest-neighbor hopping.

The corresponding $Z$ factors, plotted in Fig. 4, are also enhanced close to the surface in the presence of an electric field. In contrast to what is observed for the density, the $Z$ factor is enhanced close to the surface even at the smallest nonzero value of the applied field which we consider here $(V=1.5)$. In most cases, the highest value of $Z$ in the slab occurs not immediately at the surface but one layer into the slab. Deeper into the slab $Z$ decreases from that maximum value. At the highest temperature in the series $(\beta t=10)$ there is only a narrow central region where the enhancement is not significant. The width of the region where $Z$ is small increases as the temperature is lowered.

$A(\omega=0)$, the spectral weight at the Fermi level, is plotted in Fig. 5 for the same simulation parameters as in Figs. 3 and 4. Similarly to the $Z$ factor, $A(\omega=0)$ is enhanced most strongly close to the surfaces. In contrast to what is observed for the $Z$ factor, however, the enhancement of $A(\omega=0)$ at the lowest nonzero bias $(V=1.5)$ is insignificant. Furthermore, except at $\beta t=10$, at large enough bias, the value of $A(\omega=0)$ remains nearly constant (close to its maximum value in the slab) in the layers immediately near the surface. The transition to the low spectral weight region in the center of the slab is relatively abrupt, compared with what we observe for the $Z$ factor. Contrary to the width of the region where $Z$ is small, the width of the low spectral weight region decreases with decreasing temperature. Thus, at the highest temperature $(\beta t=10)$ there is a region of high $Z$ and low $A(\omega=0)$ close to the center of the slab, whereas at lower temperatures there is a region of strongly enhanced $A(\omega=0)$ and weakly enhanced $Z$ factor.

In combination with the charge redistribution in the slab, the field enhancement of the $Z$ factor and spectral weight at the Fermi level leads to the formation of conductive channels close to the surfaces of the slab, while the central portion of the slab remains close to its (insulating) $V=0$ state.

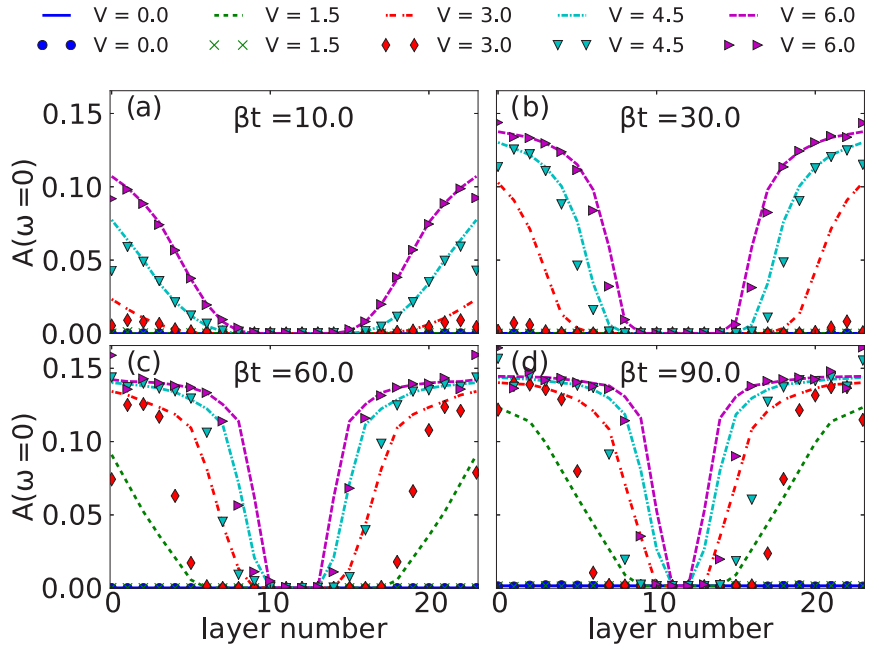

FIG. 5. The variation of the spectral weight at the Fermi level across the slab corresponding to the density and $Z$-factor data shown in Figs. 3 and 4. Symbols correspond to the full DMFT calculation, lines correspond to the LDA.

The fact that a certain minimal field is necessary to achieve a "breakdown" of the insulator (i.e., to create an electrically doped region close to the surface in which the $Z$ factor and the spectral weight at the Fermi level are significant) can be understood more easily by considering the dependence of density on chemical potential in the three-dimensional Hubbard model (Fig. 1). Due to the presence of a gap in the spectrum for large enough $U$, a plateau analogous to the one in Fig. 1 appears in the $n$ vs layer curves in Fig. 3, which corresponds to values of the local chemical potential that are well within the gap. When the externally applied field is large enough, the local value of the chemical potential close to the surface of the slab deviates sufficiently from $U / 2$ to induce a change in the local charge density.

Some of our simulations (not shown) also indicate that when $\beta t \geqslant 30$ and the applied external field is small, only an insulating solution exists in the slab at $U / t=13.2$. In other words, independent of the seed, the simulation converges to the insulating solution throughout the slab. Above a certain value of the field $(V \approx 3.0)$, two solutions reappear. This is consistent with what we observe in bulk (Fig. 1).

The temperature dependence of the bulk $n(\mu)$ curves also elucidates what is observed in the slab. At $U / t=13.2$ the range of $\mu$ values for which both a metallic and an insulating solution exist is clearly larger at lower temperature, extending to values of $\mu$ closer to the middle of the gap. On the metallic branch (Fig. 1), the plateau in $n(\mu)$ shrinks as temperature drops and almost completely disappears at $\beta=90$. The insulating solution, in contrast, is less temperature sensitive. This temperature dependence of the bulk metallic solution is consistent with what we observe in the slab (see Fig. 3): The minimum field strength required to "break" the insulator decreases as the temperature drops. The width of the insulating region that remains at half filling in the center of the slab for a given field also decreases as the temperature is lowered. Similarly to what is observed in bulk, changes in the local value of the chemical potential in individual layers in the slab 


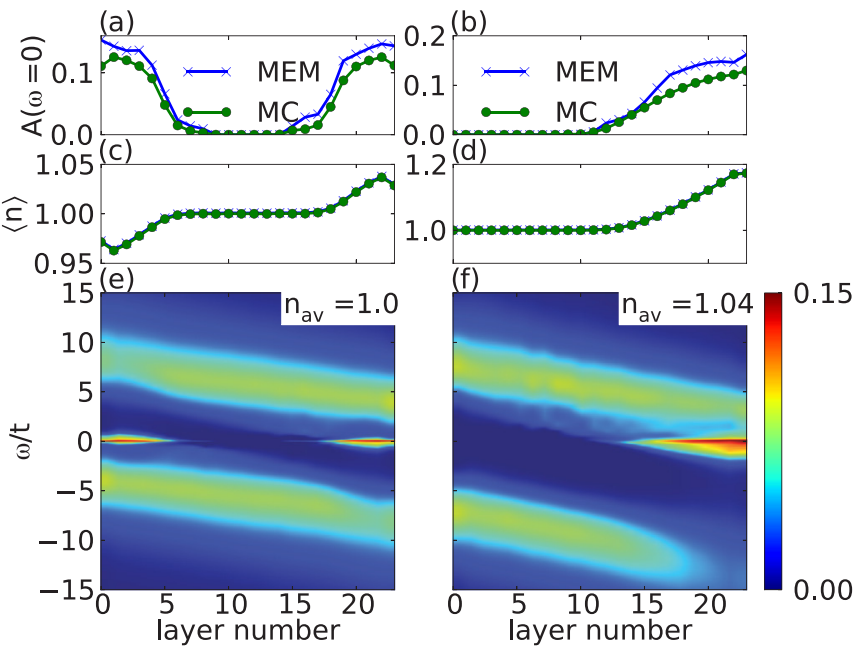

FIG. 6. Panels (e) and (f): Maximum-entropy method reconstruction of the spectral functions $A_{i}(\omega)$ throughout the slab. Data is interpolated between layers for clarity. Panels (a) and (b): Comparison of MEM results for $A(\omega=0)$ (blue lines with crosses) with those obtained by Eq. (6) (green lines with circles). Panels (c) and (d): a comparison of Eq. (9) (blue lines with crosses) and the Monte Carlo results (green lines with circles) for the density $n$. Left panels: $U / t=13.2, \mu=U / 2, \beta t=30, V=4.5$ and average density corresponding to half filling. Right panels: $U / t=16.2, \beta t=$ $10, V=6$ and average density per site in the slab $n=1.04$.

do not lead to a significant change in density, unless the change is large enough.

Maximum-entropy method (MEM) reconstructions [58,59] of the real-frequency spectral function $A(\omega)$ provide additional insight into the changes that occur across the slab as bias is increased and parts of the slab become metallic [see Fig. 6(e)]. We validated our MEM results in two different ways: Comparison with the results of Eq. (6) reveals very good agreement [Fig. 6(a)]. Furthermore, calculating the average density in each layer from the real frequency spectral function, according to:

$$
n_{i}=\int_{-\infty}^{+\infty} d \omega A_{i}(\omega) f\left(\omega-\mu_{i}\right),
$$

where $A_{i}(\omega)$ is the MEM spectral function of layer $i$ and $f(\omega)$ is the Fermi-Dirac distribution function, we find excellent agreement with the Monte-Carlo charge density data [Fig. 6(c)]. The appearance and development of the quasiparticle peaks due to the spectral transfer from the nearby Hubbard bands (the frequency-integrated spectrum remains constant) is clearly visible in the figure. The width of the peaks is proportional to the $Z$ factor and reaches a maximum approximately one layer into the slab from each of the surfaces, which is consistent with Fig. 4. Similarly, the abrupt rise of $A(\omega=0)$ is reproduced [in the MEM plot $A(\omega=0)$ corresponds to the height of the quasiparticle peak]. The MEM results for the doped case [Figs. 6(b), 6(d), 6(f)] are discussed in more detail in the following section.

It is clear (Fig. 3) that the LDA estimate of the density agrees fairly well with the full calculation for almost all the temperatures and field strengths we consider. The difference between the two approaches is greatest close to the surfaces of the slab, where the independent plane approximation tends to overestimate the density and fails to capture the dip evident in the full calculation, and for values of $V$ which are close to the "breakdown" bias of the insulator. The LDA underestimates the value of the breakdown bias. The differences between the LDA estimates for $Z$ and $A(0)$ and the full calculation are more pronounced. The largest discrepancy in $Z$ is observed at $V=$ 3 , the bias value at which the system is closest to the breakdown point. At $V=3$ and $\beta t \geqslant 30$ the LDA and full DMFT results for $Z$ differ not only near the surface, but through a significant part of the slab. A similar observation is valid for $A(\omega=0)$, but in addition to the significant disagreement between the LDA and DMFT estimates of $A(\omega=0)$ at $V=3$, the LDA also significantly overestimates the value of $A(\omega=0)$ at $V=1.5$ and $\beta t=60,90$. The agreement is better at larger values of $V$ and at higher temperature (lower $\beta$ ).

\section{Screening}

We also investigate the effect of screening on the half-filled slab (see Fig. 7). In Figs. 7(a) and 7(c) we show the density and potential across a slab in the metallic phase $(U / t=10$ and $\beta t=10)$ in the presence of screening and in the unscreened case. For the screened case we use $\alpha=1$. The total potential drop across the slab in both cases is the same (this means that in the screened case the applied external field $V$ is larger). In practice, we first fix the externally applied field $V$ and the screening strength $\alpha$ and allow the simulation to converge to a self-consistent solution that satisfies Eq. (3). This leads to a reduced potential drop across the slab. Then a nonscreened simulation with the same potential drop is performed for comparison. The effect of screening is to reduce the penetration of the field in the slab. The density deviation (from half filling) is reduced compared to the nonscreened case, especially in the central region of the slab. Qualitatively the results remain very similar: The deviation from half filling is symmetric with respect to the central region and largest close to the two surfaces in the slab. Screening masks the influence of the surface on the density: in the absence of screening the maximum of the density does not occur at the surface but one layer into the slab. In contrast, in the screened case, the maximum is at the surface. The surface reduction effect is still
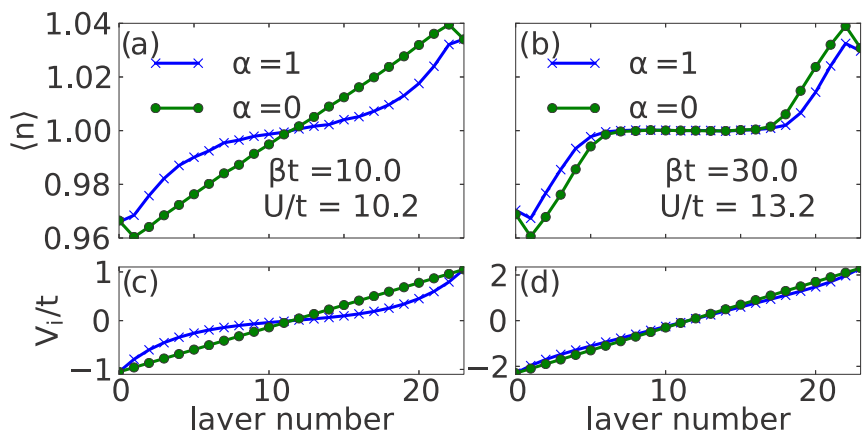

FIG. 7. The effect of screening on a metallic slab, panels (a) and (c), and an insulating slab, panels (b) and (d). The effect is much more pronounced for the metallic case. $\alpha$ is the screening parameter described in Sec. II. 
visible, but softened, due to the decreased penetration of the field in the slab. The effect of screening on an insulating slab $(U / t=13.2, \beta t=30)$ is analogous, Figs. 7(b) and 7(d). In contrast to the metallic case, however, the effect is much less pronounced. Our results are similar to the ones found earlier with the Falicov-Kimball model [44]. This indicates that the effects of Coulomb screening do not depend in an essential way on the type of the local interaction.

\section{Doped case}

The effect of the field on a doped slab is quite different. In Figs. 8-10 we show the charge density redistribution (DMFT and LDA results), $A(\omega=0)$, and $Z$ factor across the slab for four different values of $U$ and $\beta(U / t=13.2,16.2 ; \beta t=$ $10,30)$. At our chosen doping $(n=1.04)$ and in the absence of a field $(V=0)$, both the spectral weight at the Fermi level (Fig. 9) and the $Z$ factor (Fig. 10) are significant throughout the slab in all four cases, indicating a metallic phase. In this case even a small field causes charge redistribution. Since the average density in the slab is kept fixed, the pile-up of charge on one side is accompanied by a decrease in the charge density on the other side. However, if the value of $U$ is large enough (i.e., sufficient for a gap to appear in the spectral function of the solid at half filling), this decrease in the charge density on the depleted side pauses as soon as half filling is reached. From that point on, as the magnitude of $V$ increases the charge build-up on the other side is compensated by an increase in the width of the half filling region. This is what we observe here for $U / t=13.2$ and $U / t=16.2$. Thus, at large $U$, as the field increases one of the sides of the slab becomes more conducting while an insulating layer of increasing thickness develops at the opposite end of the slab. Note that the density redistribution is accompanied by a change in the $Z$ factor: the $Z$ factor is enhanced on the side with excess charge and suppressed on the

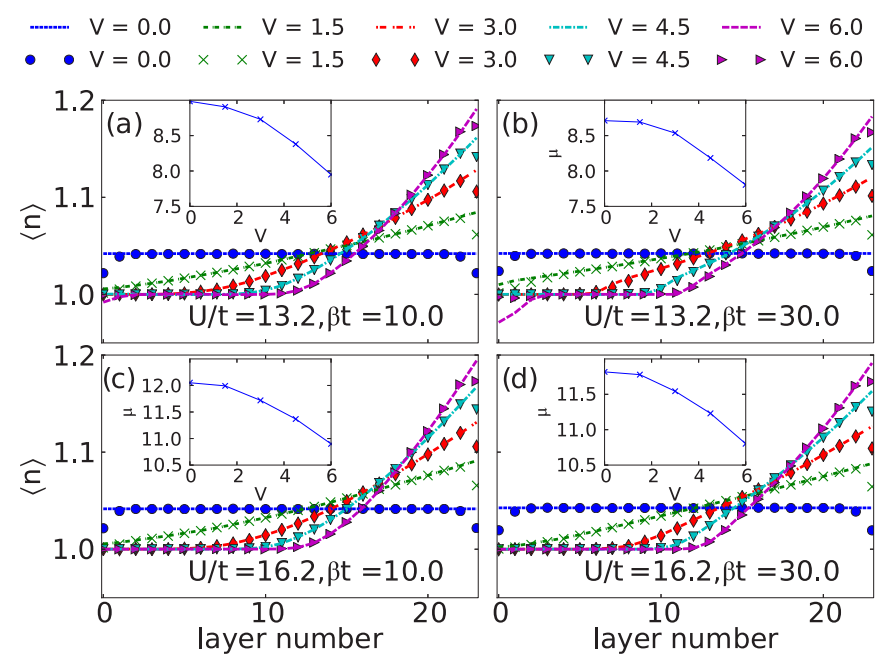

FIG. 8. Variation of the electron density throughout a 24 layer slab for selected values of the electric field, Hubbard $U$, and temperature. The average density for the whole slab is fixed at 1.04 electrons per site. The insets show how the chemical potential of the slab changes as the field is increased. This adjustment is necessary to keep the average electron density in the slab fixed. Symbols indicate the full DMFT calculation; lines correspond to the LDA.
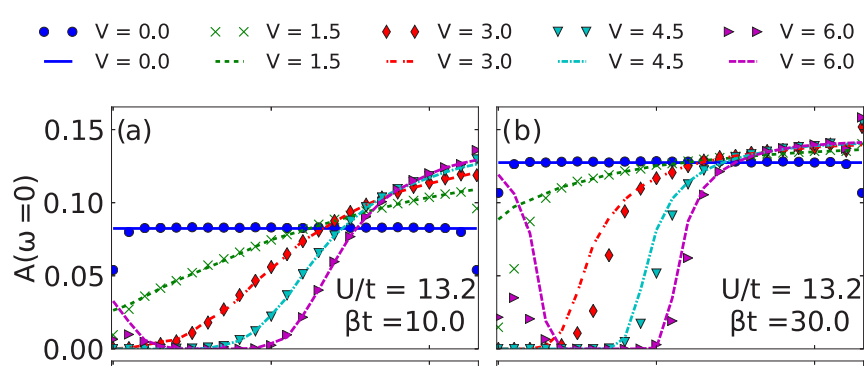

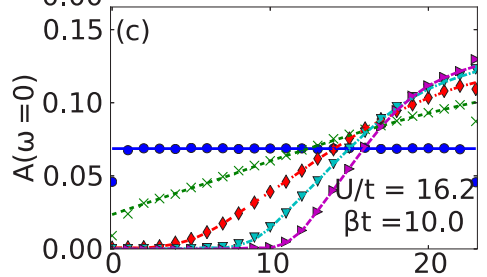

layer number

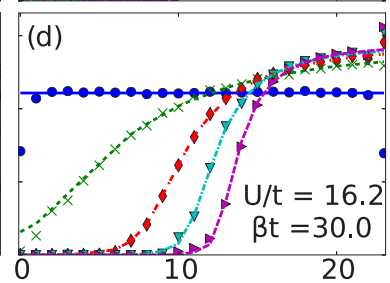

layer number
FIG. 9. Variation of the spectral weight at the Fermi level across a 24 layer slab for the same values of the electric field, Hubbard $U$, and temperature as in Fig. 8. The average density for the whole slab is fixed at 1.04 electrons per site. Symbols correspond to the full DMFT calculation, lines correspond to the LDA.

"depleted" side. The spectral weight at the Fermi level follows a similar trend. As the temperature is lowered from $\beta t=10$ to $\beta t=30$ the transition between the low- and high-conductivity regions becomes more abrupt (Fig. 9). Variation of $U / t$ in the range considered (13.2-16.2) does not significantly influence the density results. The effect of increasing $U$ on $A(\omega=0)$ is most pronounced at $V=0$, in which case it leads to a decrease of the spectral weight at the Fermi level throughout the slab.

The insets in Fig. 8 show how the chemical potential should be adjusted in order to keep the average amount of charge per layer in the slab constant (at $n=1.04$ ) at each bias. The adjustment is necessary due to the nonlinear dependence of charge density on $\mu$ (see Fig. 1). Experimentally, this situation
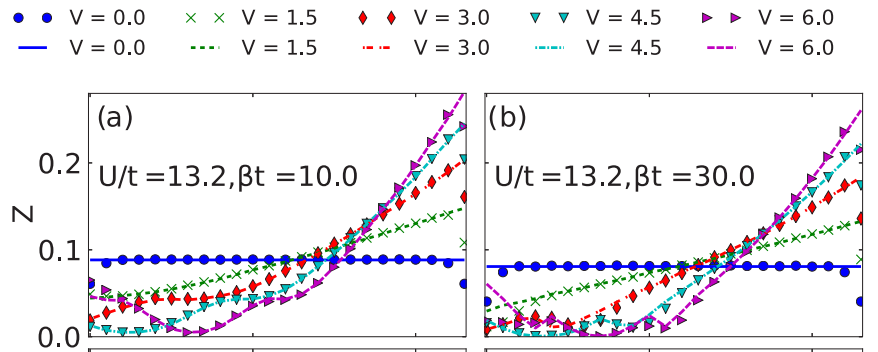

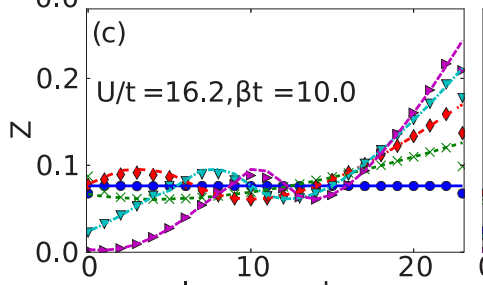

layer number

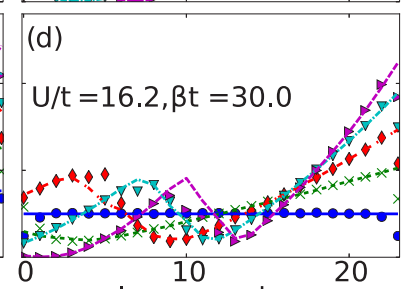

layer number
FIG. 10. Variation of the $Z$ factor across a 24 layer slab for the same values of the electric field, Hubbard $U$, and temperature as in Figs. 8 and 9. The average density for the whole slab is fixed at 1.04 electrons per site. Symbols indicate the full DMFT calculation; lines correspond to the LDA. 
corresponds to a slab that is electrically insulated from its environment (the overall amount of charge in the slab remains constant). The observed reduction of chemical potential in the presence of external electric field indicates that if connected to a charge reservoir (e.g., via metallic leads) an overdoped system would tend to absorb charge when an electric field is applied. In simulations with doped slabs at constant $\mu$ we do observe an increase in the amount of charge in the slab as the field increases (not shown). This charge absorption is caused by the nonlinearity of the $n(\mu)$ curve and not by a potential difference between the system and its environment.

At higher values of the interaction $(U / t=16.2)$ oscillations appear in the $Z$ factor vs layer number. The effect of lowering temperature is to make the oscillations more pronounced (cf. Fig. 10, lower two panels). From the fit-based estimates of the $Z$ factor (see Appendix) it is clear that the magnitude and position of these oscillations depends sensitively on the method used to estimate $Z$. In the presence of a significant spectral weight at $\omega=\mu$, these oscillations would correspond to alternating regions of low and high mobility of the quasiparticles in the film. However, since $A(\omega=0)$ is rather low in the part of the film where these oscillations occur (see Fig. 9), in practice the variation in conductivity would be small. The fact that these oscillations in $Z$ are also reproduced by the LDA indicates that they are not caused by the reduced translational symmetry in the slab geometry. It is worth noting that different parts of the slab can coexist in different states, i.e., that the metallicity of one part of the slab does not penetrate throughout the slab to destroy the low $Z$ and low spectral weight region in other parts of the slab.

The MEM reconstruction of the slab spectral functions is shown Fig. 6(f) for $U / t=16.2, \beta t=10$, and $V=6$. In this case the agreement with Eq. (6) and DMFT density data is again very good (panels (b) and (d), respectively). Due to the doping, the position of the lower and upper Hubbard bands with respect to the Fermi level is not symmetric, in contrast to the half-filled case. This asymmetry is also reflected in Fig. 8 to Fig. 10. From the MEM reconstruction it is clear that once again the $Z$ factor and spectral weight enhancement on the right side of Figs. 10 and 9 are associated with the appearance of a quasiparticle peak. On the basis of the MEM reconstruction, it is to be expected that at sufficiently strong fields the lower Hubbard band will also approach the Fermi level and a quasiparticle peak will appear on the low-doped side as well, which will cause a "secondary breakdown" to occur at the insulating side of the film (not to be confused with the oscillations in $Z$ discussed in the preceding paragraph).

The doping and the resulting asymmetry lead to a very different response compared to the half-filled case. In the latter case, the application of the field to the insulating slab leads to the formation of symmetric zones of relatively high conductivity close to the surfaces, whereas in the doped case, which is metallic in the absence of a field, application of a field causes one side to become more conductive, whereas the other side becomes insulating, until the field is large enough for the "secondary" breakdown to occur.

The LDA results for the charge density match the full calculation very nearly in almost all cases, except in the surface layers, and - when the bias is in the vicinity of the "secondary breakdown"-in a narrow region close to the surface on
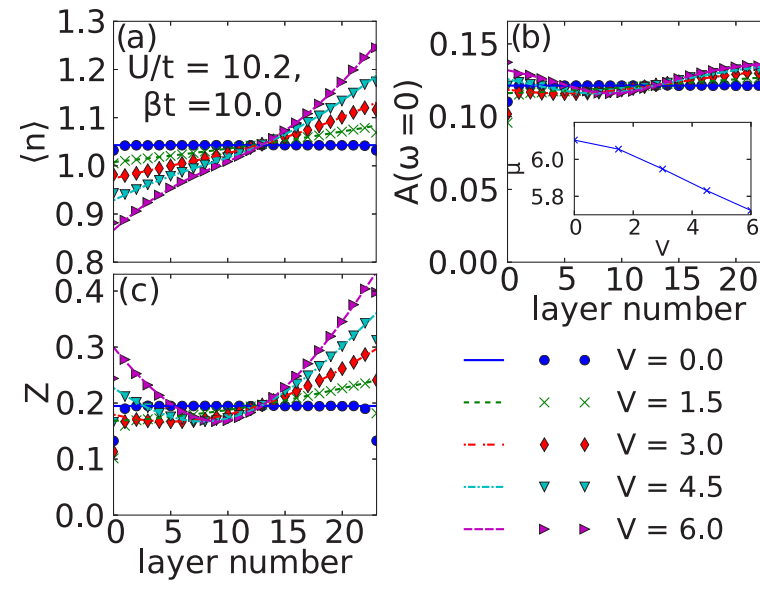

FIG. 11. Electron density, spectral weight at the Fermi level, and $Z$ factor across a doped 24 layer slab for the same values of the electric field as in Figs. 8-10, but lower $U$. The average density in the slab is fixed at 1.04 electrons per site. The inset shows the change of $\mu$ necessary to keep the average density constant for different values of $V$. Symbols correspond to the full DMFT calculation, lines correspond to the LDA.

the insulating side. The agreement on the overdoped side improves as the bias increases. We attribute this to the short correlation length in the highly-doped (metallic) regime. On the underdoped side the discrepancy is most significant at low temperature $(\beta t \geqslant 30)$, close to the "secondary breakdown." As in the half-filled case, the LDA underestimates the breakdown voltage. This tendency to overestimate the extent of the metallic phase is reflected also in the LDA results for the spectral weight, Fig. 9, and $Z$ factor, Fig. 10. Overall, the agreement between the DMFT and LDA is much better for the doped case than for the half-filled slab, which can be partially attributed to the fact that at most biases and temperatures a large part of the slab is metallic.

When the Hubbard interaction is lowered to $U / t=10.2$ (Fig. 11), no half-filling region of significant width develops, as there is no plateau in the $n(\mu)$ curve at this interaction strength. At this value of $U$ the $Z$ factor exhibits a minimum as a function of layer number and is enhanced close to the slab surfaces. Expectedly, as in the large $U$ case, even a weak field is sufficient to cause a redistribution of charge. The agreement between the DMFT and the LDA results for density, $A(\omega=0)$, and $Z$ factor is excellent at all bias values we consider. The largest discrepancy between the two approaches occurs in the two layers immediately at the surface and is most significant in the value of $Z$ [panel (c)].

\section{CONCLUSION}

In summary, we investigate the properties of strongly correlated thin films under bias using IDMFT and examine the validity of a computationally cheaper approximation. We observe switching behavior in both half-filled and doped films. In the half-filled slab a sufficiently strong field (larger than some threshold) is necessary to produce conducting regions near the surfaces of the film. For doped films, there is no threshold field and the application of a field initially causes one 
side of the film to become more insulating, before a secondary breakdown occurs. Taking a screening correction of the form given in Eq. (3) into account does not lead to qualitative changes of the results. It can therefore be expected that the conclusions for the nonscreened case will remain qualitatively valid in the presence of screening.

In spite of the breakdown of useful concepts from band theory, such as band bending, the local density approximation accurately reproduces the full IDMFT calculations in both the half-filled and doped cases, except in the layers immediately at the surface of the slab and close to transition points. Our calculations confirm and extend earlier findings in theoretical studies of colossal magnetoresistance, where DMRG calculations in 1D systems indicated the existence of a universal density-potential relation of the interface Mott transition [60]. We find that the goodness of the local approximation for $n$ and $Z$ is relatively independent of temperature in the $T$ range we explore. In contrast, the agreement between the LDA results for $A(\omega=0)$ and the full DMFT calculation is worse at lower temperature. Overall, the LDA may be used as a fairly reliable and quick first estimate for the charge density and to a lesser extent for the $Z$ factor and spectral weight at the Fermi level. This may allow for calculations of device properties such as, e.g., electrostatic charge distribution, switching behavior, differential capacitance, which are not easily accessible with full DMFT calculations due to the larger computational cost. The hysteretic behavior associated with the first order Mott transition observed in bulk persists in the slab, and should lead to memory effects in devices, which is relevant for the development of applications based on strongly-correlated materials.

\section{ACKNOWLEDGMENTS}

This work was partially funded by the Flemish Fund for Scientific Research (FWO Belgium) under FWO Grant No. G.0520.10 and the joint FWF (Austria)-FWO Grant No. GOG6616N, and by the SITOGA FP7 project. Most of the calculations were performed on KU Leuven's ThinKing HPC cluster provided by the VSC (Flemish Supercomputer Center), funded by the FWO and the Flemish Government-department EWI.

\section{APPENDIX}

For completeness, in this section we present estimates of $Z$ and $\operatorname{Im} \Sigma(\omega=0)$ based on a polynomial fit of the form:

$$
\operatorname{Im} \Sigma(i \omega)=a_{0}+a_{1} \omega+a_{2} \omega^{2}+a_{3} \omega^{3}+\cdots
$$

to the imaginary part of the self-energy at the few lowest Matsubara frequencies. In this case

$$
Z=\left.\frac{1}{1-\partial \operatorname{Im} \Sigma(i \omega) / \partial \omega}\right|_{\omega \rightarrow 0^{+}}=\frac{1}{1-a_{1}} .
$$

The $Z$-factor obtained by fitting a polynomial of the form Eq. (10) of degree four to the self-energy at the lowest six Matsubara frequencies are compared with the one based on Eq. (5) in Figs. 12 (half-filled case) and 14 (doped case). (Polynomials of order $n$ with $8 \geqslant n \geqslant 3$, fitted to the values of $\operatorname{Im} \Sigma\left(i \omega_{n}\right)$ at the lowest $n+2$ Matsubara frequencies, were considered, showing only a weak dependence on $n$.)

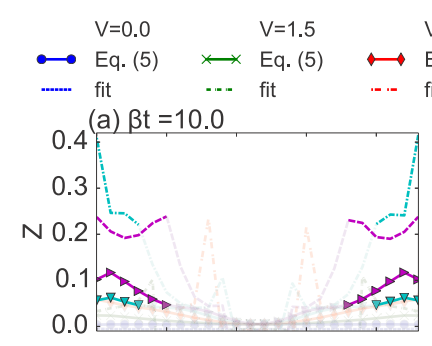

$V=3.0 \quad V=4.5$

$\mathrm{V}=6.0$
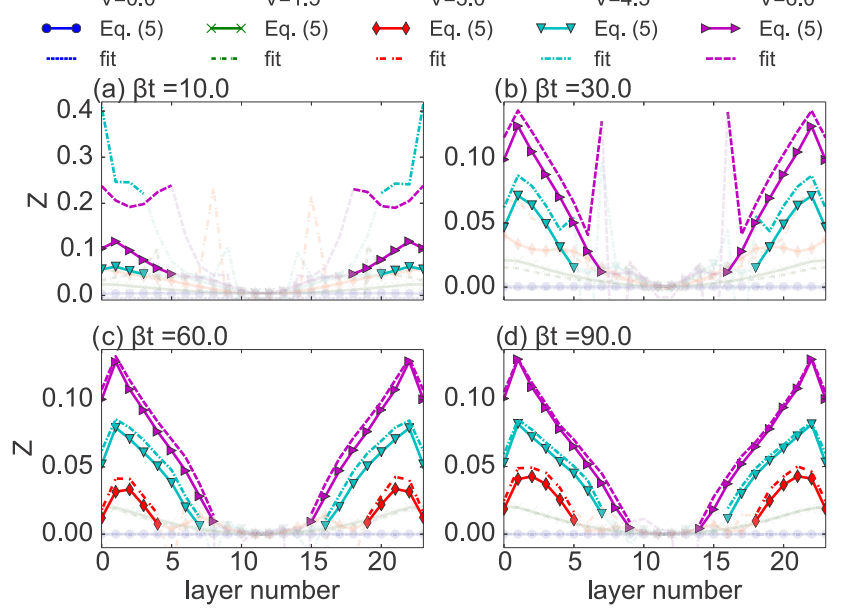

FIG. 12. Comparison of the $Z$ factor estimated from a fit, Eq. (10) (lines), and from the simple form, Eq. (5) (lines with symbols), across the slab for the same simulation parameters as in Figs. 3-5 (halffilled case, $U / t=13.2$ ). Semitransparency indicates regions with low spectral weight at the Fermi level (see the last section of the Appendix for details).

In Figs. 12 and 14 we use different opacities to distinguish between "metallic" (opaque colors) and "insulating" (semitransparent colors) regions of the slab. The rationale behind the distinction is described in the last subsection below.

\section{Half-filled case}

In Fig. 12 we show a comparison between the $Z$ factor estimated for the half-filled case on the basis of the approximate expression, Eq. (5) (lines with symbols; already shown in Fig. 4), and the fit, Eq. (10) (lines). The high-temperature case $(\beta t=10)$ is quite different from the rest, so we discuss it separately below.

At the lower temperatures considered here $(\beta t=30,60,90)$ the agreement between the fit and the simpler approximate expression is very good in the metallic regions of the slab, and it improves as the temperature decreases. In the insulating (central) regions of the slab and in the regions of transition between the metallic and insulating parts of the slab, on the other hand, the agreement is poor even qualitatively. Whereas the simple approximation, Eq. (5), always yields a positive $Z$ factor of magnitude less than 1 [as long as $\operatorname{Im} \Sigma\left(i \omega_{0}\right)$ is negative, which is always the case here], the fit can result in a positive slope of the imaginary part of the self-energy at small Matsubara frequency $\left(a_{1}>0\right)$, which-depending on whether $a_{1}>1$ or $a_{1}<1$ - can result in $Z<0$ or $Z>1$, respectively. The former case, $Z<0$, occurs in the central region of the half-filled slabs, where we find $a_{1} \gg 1$ at all temperatures considered here. The latter case, $Z>1$, occurs in the transition regions between the metallic and insulating portions of the slab, where the sign of $a_{1}$ changes from negative to positive. This case causes the appearance of the spikes at, e.g., $V=6, \beta=30$ [Fig. 12(b)].

It is clear that in the high-temperature case $[\beta t=10$, Fig. 12(a)] the agreement between the fit-based estimate for $Z$ and the naive expression is rather poor. This is also reflected in 

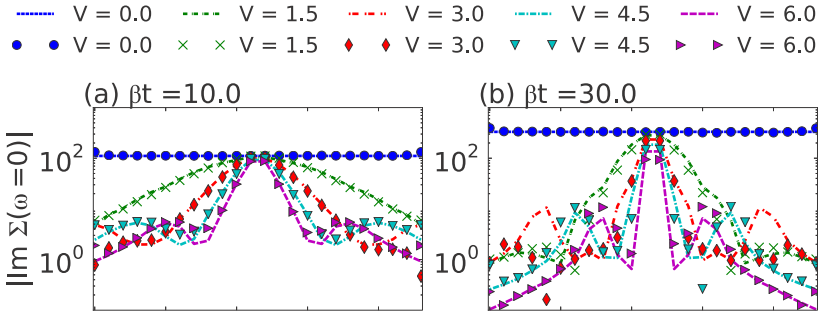

$$
\text { (c) } \beta \mathrm{t}=60.0
$$

(d) $\beta \mathrm{t}=90.0$
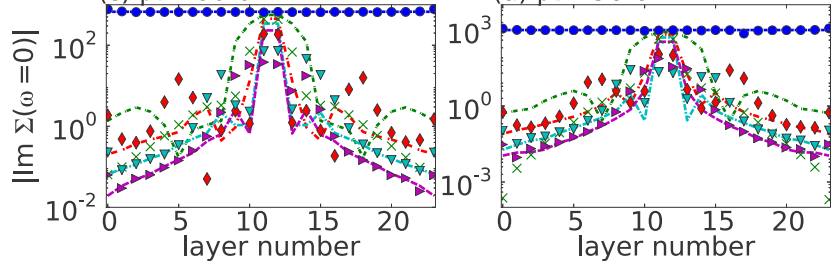

FIG. 13. Variation of the absolute value of $\operatorname{Im} \Sigma(\omega=0)$ (estimated from a fit to the imaginary part of the self-energy) across the slab for the same simulation parameters as in Figs. 3-5 and Fig. 12 (half-filled case, $U / t=13.2$ ). Symbols correspond to the full DMFT calculation, lines correspond to the LDA.

the fact that the magnitude of $\operatorname{Im} \Sigma(\omega=0)$ at all biases (except $V=0)$ is significantly larger than at the respective biases at all other temperatures [cf. Figs. 13(a) and 13(b)-13(d)]: $\operatorname{Im} \Sigma(\omega=0) \gtrsim 1$ throughout the slab at $\beta=10$ whereas at lower temperatures $\operatorname{Im} \Sigma(\omega=0)$ is generally $\lesssim 1$, except in a few central layers. The large value of $\operatorname{Im} \Sigma(\omega=0)$ indicates a shortened quasiparticle lifetime, which means that at this temperature excitations near the Fermi level are far less coherent than at the lower temperatures considered. In the absence of coherent excitations (the "bad metal" regime [61]) the $Z$ factor cannot properly be interpreted as a quasiparticle mass renormalization factor (irrespective of whether it is calculated from the fit to the self-energy or the more naive expression). For these reasons it is clear that a quantitative comparison between the $\beta t=10$ case and the lower-temperature cases is not meaningful. The trends in Fig. 13(a) are nevertheless clear: Under bias, quasiparticle excitations near the center of the slab are much less coherent than close to the surfaces.

\section{Doped case}

In the doped case, we see again that at high temperature [ $\beta t=10$, Figs. 14(a) and 14(c)] there is a large discrepancy between the fit-based estimate of $Z$ and the simpler approximation. Also similar to the half-filled case is the observation that the magnitude of $\operatorname{Im} \Sigma(\omega=0)$ at high temperature is about an order of magnitude larger than at the lower temperature we consider here $(\beta t=30)$ throughout most of the slab (cf. Fig. 15). For the same reasons as in the half-filled case, we ascribe the poor agreement between the fit and the approximate expression, Eq. (5), to the lack of coherent excitations at this temperature. This means that the variation of the $Z$ factor at this temperature doesn't provide useful information about the conductivity of the different layers of the slab. As in the half-filled case, however, the variation of $\operatorname{Im} \Sigma(\omega=0)$ [cf. Figs. 15(a) and 15(c)] allows us some insight into how conductivity changes across the slab. It is clear that the overall conclusions made on the basis of the approximate expression,

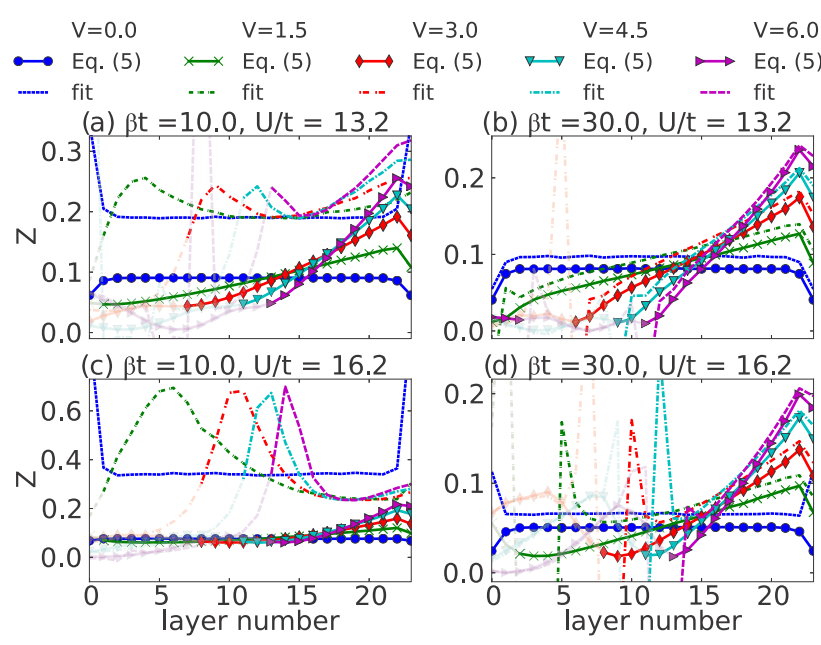

FIG. 14. Comparison of the $Z$ factor estimated from a fit, Eq. (10) (lines), and from the simple form, Eq. (5) (lines with symbols), across the slab for the same simulation parameters as in Figs. 8-10 (doped case). Semitransparency indicates regions with low spectral weight at the Fermi level (see the last section of the Appendix for details).

Eq. (5), remain valid: Under bias, one side of the slab becomes more conducting, whereas the other becomes more insulating.

At lower temperature [ $\beta t=30$, Figs. $14(\mathrm{~b})$ and $14(\mathrm{~d})]$, we also encounter a situation similar to the half-filled case: The agreement between the fit, Eq. (10), and Eq. (5) is good, except in those areas of the slab which are insulating. In particular, the oscillations that appear in $Z$ when Eq. (5) is used are not reproduced by the fit.

The suppression of $Z$ at the surface observed when Eq. (5) is used is reproduced by the fit, with the exception of the $V=0, U / t=16$ case [Fig. 14(d)], which therefore deserves additional comment. The slight enhancement of $Z$ at the surface $(\approx 40 \%$ with respect to bulk) in this case is accompanied by a much larger enhancement (nearly tenfold
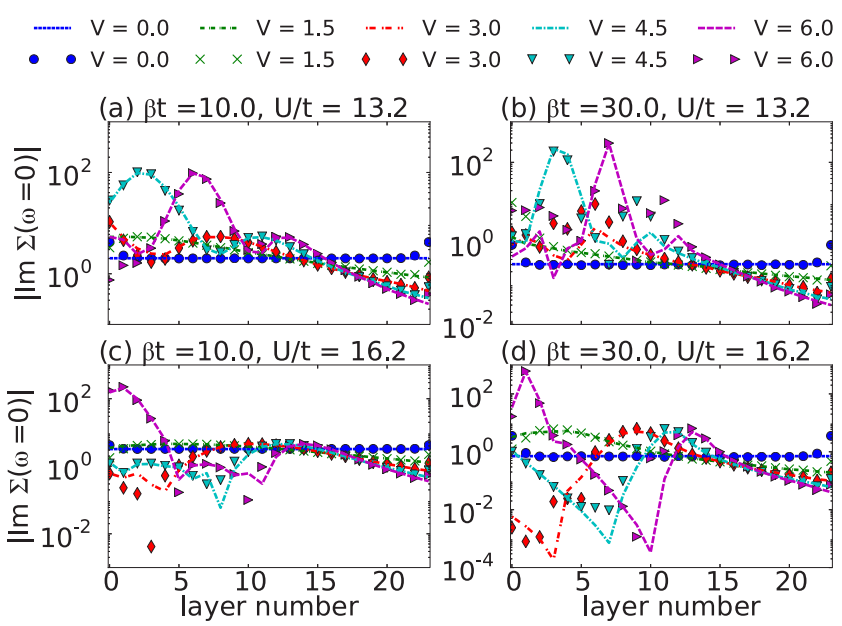

FIG. 15. Variation of the absolute value of $\operatorname{Im} \Sigma(\omega=0)$ (estimated from a fit to the imaginary part of the self-energy) across the slab for the same simulation parameters as in Figs. 8 and 9 and Fig. 14 (doped case). Symbols correspond to the full DMFT calculation, lines correspond to the LDA. 
with respect to the bulk value) of $\operatorname{Im} \Sigma(\omega=0)$ (cf. Fig. 15). As the magnitude of $\operatorname{Im} \Sigma(0)$ is inversely proportional to the quasiparticle lifetime, this means that the earlier conclusion that the surface layers are more insulating remains valid also in this case.

\section{Quality of the fit}

We also estimated the goodness of the fits used to obtain the data shown in Figs. 12-15. We did this by estimating the $Q$ value, i.e., the likelihood that $\chi^{2}$ will exceed a given value by chance, of each of the fits (for more details on goodness-offit estimation see, e.g., Numerical Recipes, chapter 15 [62]). Except in the strongly insulating regions of the slab, the value of $Q$ is $\approx 10^{-1}$ In the strongly insulating regions of the slab the value of $Q$ is extremely small $\left(\left\langle 10^{-14}\right.\right.$ in some layers). In general, a fit probability $Q>0.001$ indicates a reasonably good fit to the data. [62] Thus the extremely small $Q$ values we see in some cases indicate that the functional form Eq. (10) is a very unlikely model for the behavior of $\operatorname{Im} \Sigma\left(i \omega_{n}\right)$ in those cases. A fit with a $1 / \omega$ term provides a much higher $Q$ value $(\approx 0.1)$ in those cases (the overall number parameters being kept fixed). This is not surprising, as $\operatorname{Im} \Sigma(\omega)$ is expected to diverge at $\omega=0$ in the Mott insulating phase.

\section{4. $\operatorname{Im} G\left(i \omega_{n}\right)$ criterion}

For clarity in Figs. 12 and 14 we use different opacities to distinguish between "metallic" and "insulating" regions of the slab. In this section we describe the criterion which was used for that distinction.

The basis for the distinction is the observation that the shape of $\operatorname{Im} G\left(i \omega_{n}\right)$ in insulating layers is different from that in metallic layers. This is illustrated in Fig. 16, which shows how $\operatorname{Im} G\left(i \omega_{n}\right)$ changes across a slab under bias (in this case $U / t=13.2, \beta t=30, V=6$ ). Deep in the metallic

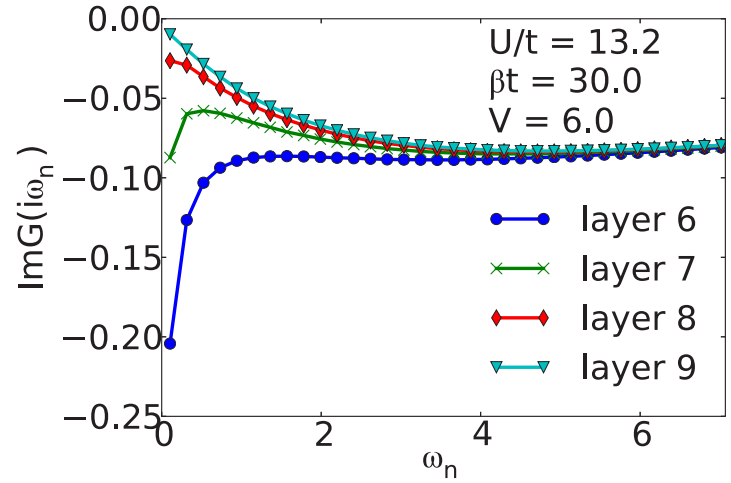

FIG. 16. A plot of the imaginary part of $G\left(i \omega_{n}\right)$ for different layers in the slab illustrating the criterion used to distinguish between "metallic" and "insulating" parts of the slab (see text).

region $\operatorname{Im} G\left(i \omega_{n}\right)$ exhibits a negative peak close to the origin [the slope of $\operatorname{Im} G\left(i \omega_{n}\right)$ is positive close to the origin] and attains its lowest value at the first Matsubara frequency. In the strongly insulating layers, on the other hand, $\operatorname{Im} G\left(i \omega_{n}\right)$ has negative slope and extrapolates nearly to zero at the origin. The transition between the two extremes is fairly abrupt $(\approx 3$ layers in most cases). We use as our "cutoff" between "metallic" and "insulating" layers the point where the $\operatorname{Im} G\left(i \omega_{n}\right)$ no longer attains its lowest value at the lowest Matsubara frequency $i \omega_{0}$ [63]. Obviously there is a certain degree of arbitrariness in labeling the two neighboring layers on either side of that cutoff as "metallic" and "insulating," respectively, as in fact they are fairly similar.

It is worth pointing out that the point where this change of slope occurs is closely correlated with the point where $A(\omega=0)$, the spectral weight at the Fermi level, exhibits a sharp drop (see Figs. 5 and 9). This isn't surprising, since $A(\omega=0)=-\operatorname{Im} G(\omega=0) / \pi$.
[1] R. W. Helmes, T. A. Costi, and A. Rosch, Phys. Rev. Lett. 101, 066802 (2008).

[2] H. Zenia, J. K. Freericks, H. R. Krishnamurthy, and T. Pruschke, Phys. Rev. Lett. 103, 116402 (2009).

[3] J. M. Tomczak, F. Aryasetiawan, and S. Biermann, Phys. Rev. B 78, 115103 (2008).

[4] K. Martens, I. P. Radu, S. Mertens, X. Shi, L. Nyns, S. Cosemans, P. Favia, H. Bender, T. Conard, M. Schaekers, S. De Gendt, V. Afanas'ev, J. A. Kittl, M. Heyns, and M. Jurczak, J. Appl. Phys. 112, 124501 (2012).

[5] J. Jeong, N. Aetukuri, T. Graf, T. D. Schladt, M. G. Samant, and S. S. P. Parkin, Science 339, 1402 (2013).

[6] K. Martens, N. Aetukuri, J. Jeong, M. G. Samant, and S. S. P. Parkin, Appl. Phys. Lett. 104, 081918 (2014).

[7] M. Nakano, K. Shibuya, D. Okuyama, T. Hatano, S. Ono, M. Kawasaki, Y. Iwasa, and Y. Tokura, Nature (London) 487, 459 (2012).

[8] A. D. Caviglia, S. Gariglio, N. Reyren, D. Jaccard, T. Schneider, M. Gabay, S. Thiel, G. Hammerl, J. Mannhart, and J.-M. Triscone, Nature (London) 456, 624 (2008).
[9] M. Imada, A. Fujimori, and Y. Tokura, Rev. Mod. Phys. 70, 1039 (1998).

[10] P. Limelette, A. Georges, D. Jérome, P. Wzietek, P. Metcalf, and J. M. Honig, Science 302, 89 (2003).

[11] S. Okamoto and A. J. Millis, Nature (London) 428, 630 (2004).

[12] H. Y. Hwang, Y. Iwasa, M. Kawasaki, B. Keimer, N. Nagaosa, and Y. Tokura, Nat. Mater. 11, 103 (2012).

[13] G. Kotliar, S. Y. Savrasov, K. Haule, V. S. Oudovenko, O. Parcollet, and C. A. Marianetti, Rev. Mod. Phys. 78, 865 (2006).

[14] M. C. Gutzwiller, Phys. Rev. Lett. 10, 159 (1963).

[15] J. Bünemann, W. Weber, and F. Gebhard, Phys. Rev. B 57, 6896 (1998).

[16] F. Gebhard, Phys. Rev. B 41, 9452 (1990).

[17] G. Kotliar and A. E. Ruckenstein, Phys. Rev. Lett. 57, 1362 (1986).

[18] F. Lechermann, A. Georges, G. Kotliar, and O. Parcollet, Phys. Rev. B 76, 155102 (2007).

[19] A. Rüegg, S. Pilgram, and M. Sigrist, Phys. Rev. B 75, 195117 (2007). 
[20] G. Borghi, M. Fabrizio, and E. Tosatti, Phys. Rev. B 81, 115134 (2010).

[21] D. Nasr Esfahani, L. Covaci, and F. M. Peeters, Phys. Rev. B 85, 085110 (2012).

[22] D. N. Nasr Esfahani, L. Covaci, and F. M. Peeters, Phys. Rev. B 87, 035131 (2013).

[23] D. N. Esfahani, L. Covaci, and F. M. Peeters, J. Phys.: Condens. Matter 26, 075601 (2014).

[24] Y. Omori, A. Rüegg, and M. Sigrist, Phys. Rev. B 90, 155118 (2014).

[25] R. Raimondi and C. Castellani, Phys. Rev. B 48, 11453 (1993).

[26] M. Ganahl, M. Aichhorn, P. Thunström, K. Held, H. G. Evertz, and F. Verstraete, Phys. Rev. B 92, 155132 (2015).

[27] A. Georges, G. Kotliar, W. Krauth, and M. J. Rozenberg, Rev. Mod. Phys. 68, 13 (1996).

[28] W. Metzner and D. Vollhardt, Phys. Rev. Lett. 62, 324 (1989).

[29] J. Hubbard, Proc. R. Soc. London A 276, 238 (1963).

[30] P. Anderson, Phys. Rev. 124, 41 (1961).

[31] T. Maier, M. Jarrell, T. Pruschke, and M. H. Hettler, Rev. Mod. Phys. 77, 1027 (2005).

[32] K. Held, Adv. Phys. 56, 829 (2007).

[33] M. Potthoff and W. Nolting, Phys. Rev. B 59, 2549 (1999).

[34] M. Potthoff and W. Nolting, Phys. Rev. B 60, 7834 (1999).

[35] J. K. Freericks, B. K. Nikolić, and P. Miller, Phys. Rev. B 64, 054511 (2001).

[36] S. Okamoto and A. J. Millis, Phys. Rev. B 70, 241104 (2004).

[37] S. Okamoto and A. J. Millis, Phys. Rev. B 72, 235108 (2005).

[38] C. Lin, S. Okamoto, and A. J. Millis, Phys. Rev. B 73, 041104 (2006).

[39] W.-C. Lee and A. H. MacDonald, Phys. Rev. B 74, 075106 (2006).

[40] S. Okamoto, Phys. Rev. B 76, 035105 (2007).

[41] S. Okamoto, Phys. Rev. Lett. 101, 116807 (2008).

[42] S. Okamoto and T. A. Maier, Phys. Rev. Lett. 101, 156401 (2008).

[43] C. Lin and A. J. Millis, Phys. Rev. B 78, 184405 (2008).

[44] S. T. F. Hale and J. K. Freericks, Phys. Rev. B 85, 205444 (2012).

[45] H. Ishida and A. Liebsch, Phys. Rev. B 79, 045130 (2009).
[46] M. Charlebois, S. R. Hassan, R. Karan, D. Sénéchal, and A.-M. S. Tremblay, Phys. Rev. B 87, 035137 (2013).

[47] P. Bakalov, B. Ydens, and J.-P. Locquet, Phys. Status Solidi (a) 211, 440 (2014).

[48] J. K. Freericks, Phys. Rev. B 70, 195342 (2004).

[49] Z. Zhong, M. Wallerberger, J. M. Tomczak, C. Taranto, N. Parragh, A. Toschi, G. Sangiovanni, and K. Held, Phys. Rev. Lett. 114, 246401 (2015).

[50] “Triqs website," http://ipht.cea.fr/triqs/, accessed: 2014-09-30.

[51] P. Werner, A. Comanac, L. de' Medici, M. Troyer, and A. J. Millis, Phys. Rev. Lett. 97, 076405 (2006).

[52] P. Werner and A. J. Millis, Phys. Rev. B 74, 155107 (2006).

[53] L. Boehnke, H. Hafermann, M. Ferrero, F. Lechermann, and O. Parcollet, Phys. Rev. B 84, 075145 (2011).

[54] S. Fuchs, E. Gull, M. Troyer, M. Jarrell, and T. Pruschke, Phys. Rev. B 83, 235113 (2011).

[55] H. Kajueter, G. Kotliar, and G. Moeller, Phys. Rev. B 53, 16214 (1996).

[56] P. Werner and A. J. Millis, Phys. Rev. B 75, 085108 (2007).

[57] M. Potthoff and W. Nolting, Eur. Phys. J. B 8, 555 (1999).

[58] A. W. Sandvik, Phys. Rev. B 57, 10287 (1998).

[59] M. Jarrell and J. Gubernatis, Phys. Rep. 269, 133 (1996).

[60] T. Oka and N. Nagaosa, Phys. Rev. Lett. 95, 266403 (2005).

[61] For a brief discussion of the criteria for a metal to qualify as "bad" see,e.g., V. J. Emery and S. A. Kivelson, Phys. Rev. Lett. 74, 3253 (1995).

[62] W. H. Press, S. A. Teukolsky, W. T. Vetterling, and B. P. Flannery, Numerical Recipes in Fortran 77. The Art of Scientific Computing, 2nd ed., Volume 1 of Fortran Numerical Recipes (Cambridge University Press, Cambridge, UK, 1992).

[63] We also investigated an alternative cutoff: the point where the slope of $\operatorname{Im} G\left(i \omega_{n}\right)$ at the lowest Matsubara frequency changes from positive to negative. It turns out that the two lead to very similar results in all the cases we considered, with a difference in the position of the boundary between metal and insulator of at most two layers. In all cases in which a difference existed between the two approaches, the one based on the derivative tended to favor the metallic phase, i.e., to yield a wider metallic region. 